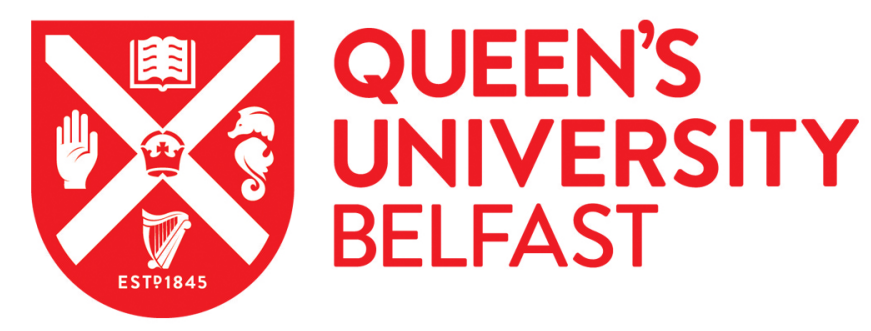

\title{
The magnetic properties of photospheric magnetic bright points with high-resolution spectropolarimetry
}

Keys, P. H., Reid, A., Mathioudakis, M., Shelyag, S., Henriques, V. M. J., Hewitt, R. L., Del Moro, D., Jafarzadeh, S., Jess, D. B., \& Stangalini, M. (2019). The magnetic properties of photospheric magnetic bright points with high-resolution spectropolarimetry. Monthly Notices of the Royal Astronomical Society: Letters, 488(1), L53-L58. https://doi.org/10.1093/mnrasl/slz097

Published in:

Monthly Notices of the Royal Astronomical Society: Letters

Document Version:

Peer reviewed version

Queen's University Belfast - Research Portal:

Link to publication record in Queen's University Belfast Research Portal

Publisher rights

(C) 2019 The Author(s)

Published by Oxford University Press on behalf of the Royal Astronomical Society.

This work is made available online in accordance with the publisher's policies. Please refer to any applicable terms of use of the publisher.

\section{General rights}

Copyright for the publications made accessible via the Queen's University Belfast Research Portal is retained by the author(s) and / or other copyright owners and it is a condition of accessing these publications that users recognise and abide by the legal requirements associated with these rights.

Take down policy

The Research Portal is Queen's institutional repository that provides access to Queen's research output. Every effort has been made to ensure that content in the Research Portal does not infringe any person's rights, or applicable UK laws. If you discover content in the Research Portal that you believe breaches copyright or violates any law, please contact openaccess@qub.ac.uk. 


\title{
The magnetic properties of photospheric magnetic bright points with high-resolution spectropolarimetry
}

\author{
Peter H. Keys ${ }^{\oplus},{ }^{1 \star}$ Aaron Reid ${ }^{\odot}, 1$ Mihalis Mathioudakis ${ }^{\oplus},{ }^{1}$ Sergiy Shelyag ${ }^{\odot},{ }^{2}$ Vasco \\ M. J. Henriques ${ }^{\odot}, 3,4$ Rebecca L. Hewitt, ${ }^{1}$ Dario Del Moro ${ }^{\odot},{ }^{5}$ Shahin Jafarzadeh ${ }^{\odot}, 3,4$ \\ David B. Jess $^{\oplus 1,6}$ and Marco Stangalini ${ }^{\oplus 7,8}$ \\ ${ }^{1}$ Astrophysics Research Centre, School of Mathematics and Physics, Queen's University Belfast, Belfast BT7 1NN, Northern Ireland, UK \\ ${ }^{2}$ School of Information Technology, Faculty of Science, Engineering and Built Environment, Deakin University, Geelong, Victoria, VIC 3125, Australia \\ ${ }^{3}$ Institute of Theoretical Astrophysics, University of Oslo, P.O. Box 1029 Blindern, NO-0315 Oslo, Norway \\ ${ }^{4}$ Rosseland Centre for Solar Physics, University of Oslo, P.O. Box 1029 Blindern, N-0315 Oslo, Norway \\ ${ }^{5}$ Dipartimento di Fisica, Università degli Studi di Roma 'Tor Vergata', via della Ricerca Scientifica 1, I-00133 Roma, Italy \\ ${ }^{6}$ Department of Physics and Astronomy, California State University Northridge, Northridge, CA 91330, USA \\ ${ }^{7}$ INAF - OAR National Institute for Astrophysics, Via Frascati 33, I-00078 Monte Porzio Catone (RM), Italy \\ ${ }^{8}$ ASI Agenzia Spaziale Italiana, Via del Politecnico snc, I-00133 Rome, Italy
}

Accepted 2019 June 13. Received 2019 June 13; in original form 2019 April 5

\begin{abstract}
Magnetic bright points (MBPs) are small-scale magnetic elements ubiquitous across the solar disc, with the prevailing theory suggesting that they form due to the process of convective collapse. Employing a unique full Stokes spectropolarimetric data set of a quiet Sun region close to disc centre obtained with the Swedish Solar Telescope, we look at general trends in the properties of magnetic bright points. In total we track 300 MBPs in the data set and we employ NICOLE inversions to ascertain various parameters for the bright points such as line-of-sight magnetic field strength and line-of-sight velocity, for comparison. We observe a bimodal distribution in terms of maximum magnetic field strength in the bright points with peaks at $\sim 480 \mathrm{G}$ and $\sim 1700 \mathrm{G}$, although we cannot attribute the kilogauss fields in this distribution solely to the process of convective collapse. Analysis of MURAM simulations does not return the same bimodal distribution. However, the simulations provide strong evidence that the emergence of new flux and diffusion of this new flux play a significant role in generating the weak bright point distribution seen in our observations.
\end{abstract}

Key words: Sun: activity -Sun: evolution-Sun: photosphere.

\section{INTRODUCTION}

Magnetic bright points (MBPs) were first observed in the late 1970s (Dunn \& Zirker 1973) in G-band images of the photosphere. Theoretical work (Spruit 1979) then described the formation of such concentrated field strengths at small scales in a process termed 'convective collapse'. The magnetic field is advected into the intergranular lanes, where it concentrates. Fast downflows within the intergranular lanes coupled with pressure differences between the magnetic flux tubes and their surroundings result in the flux tubes 'collapsing' to a point where the field strength of the tube balances the pressure exerted on it from outside. In general, such balancing of magnetic field strengths with the gas pressure is called equipartition field strengths and within flux tubes these field strengths can reach values of the order of a kilogauss (Leighton 1963; Parker 1978;
Webb \& Roberts 1978; Spruit 1979; Bellot Rubio et al. 2001; Nagata et al. 2008). Optical depth unity within the flux tube is then deeper than the surrounding plasma and the base of the tube is then heated by the hot granular walls surrounding it. This coupled with the fact that the flux tube is partially evacuated makes it appear as a localized intensity enhancement within the intergranular lane, hence the term 'magnetic bright point'.

Nagata et al. (2008) employed Hinode observations to study the formation of a single MBP, looking for the signature fast downflows within the region prior to amplification of the magnetic field to kilogauss strengths and the associated intensity enhancement as the MBP forms. A more rigorous statistical study using similar techniques followed and showed that the radii of the 49 studied MBPs reduced on average from 0.43 arcsec to 0.35 arcsec, resulting in field strengths up to $1.65 \mathrm{kG}$ (Fischer et al. 2009).

More recent studies of the magnetic field of MBPs show that it largely displays a bimodal distribution (Utz et al. 2013). In this

^E-mail: p.keys@qub.ac.uk 
study the authors employed Hinode/SOT spectropolarimetry data to observe MBPs in four different regions (i.e. a sunspot group, near pores, and in two different quiet Sun regions). Generally, the authors report a bimodal distribution with peaks around $\sim 200-300 \mathrm{G}$ and $\sim 1100-1300 \mathrm{G}$ across the four data sets. They were able to fit the distribution with lognormal components and suggested that the two peaks are due to the convective collapse process, with the weaker group representing uncollapsed MBPs and the stronger group representing those that have undergone the convective collapse process, resulting in higher field strengths.

Within this work we use a unique data set with high spatial resolution spectropolarimetric information to ascertain the nature of magnetic field distributions in MBPs. The novel aspect here is that we employ magnetohydrodynamic (MHD) simulations as a comparison to determine the relation between the distribution and the process of convective collapse.

\section{OBSERVATIONS AND NUMERICAL SIMULATIONS}

The data employed in this study was acquired with the $1 \mathrm{~m}$ Swedish Solar Telescope (SST; Scharmer et al. 2003) on 2014 July 27 from around 14:18 UT until 15:11 UT of a quiet Sun region at disc centre, with an initial pointing of N0.14W4.5 in the heliographic coordinate system. The CRisp Imaging SpectroPolarimeter (CRISP; Scharmer 2006; Scharmer et al. 2008) was used to sample the Fe I 6301 and $6302 \AA$ line pair in full Stokes spectropolarimetry mode at 32 wavelength positions with a spectral full width at half-maximum of $53.5 \mathrm{~m} \AA$ and a step size of around $37 \mathrm{~m} \AA$ for most of the line, extending to around $77 \mathrm{~m} \AA$ at the wings. A total of 92 complete full Stokes scans were taken over the duration of the observations. The images were reconstructed using the Multi-Object Multi-Frame Blind Deconvolution technique (van Noort, Rouppe van der Voort \& Löfdahl 2005; Henriques 2012) within the CRISPRED data reduction pipeline (de la Cruz Rodríguez et al. 2015) prior to being de-rotated, aligned, and destretched, which resulted in a reduced cadence for the scans of around $33 \mathrm{~s}$. The effective field of view (FOV) of the data was approximately $50 \operatorname{arcsec} \times 50$ arcsec with a spatial sampling of 0.059 arcsec pixel $^{-1}$.

The simulations were produced using the MURAM radiative MHD code (Vögler et al. 2005). This code solves large-eddy radiative three-dimensional MHD equations on a Cartesian grid, and employs a fourth-order Runge-Kutta scheme to advance the numerical solution in time. The numerical domain has a physical size of $12 \times 12 \mathrm{Mm}^{2}$ in the horizontal direction, $1.4 \mathrm{Mm}$ in the vertical direction, and is resolved by $480 \times 480 \times 100$ grid cells, respectively. More information on the simulations can be found in previous work (Keys et al. 2011, 2013; Cegla et al. 2013). Here we employ two sets of simulations, one with an initial field strength of $200 \mathrm{G}$ and one with an initial field strength of $50 \mathrm{G}$. The simulations had an effective cadence of $\sim 17$ and $\sim 34 \mathrm{~s}$ and a duration of approximately 90 and $70 \mathrm{~min}$ of physical time for the 200 and $50 \mathrm{G}$ simulations, respectively. Fig. 1 shows a sample image from both our observational and simulated data sets.

We employed a tracking algorithm (Crockett et al. 2010) to isolate the MBPs within our observations and simulations. This algorithm is based on intensity thresholding techniques to isolate bright structures within the FOV, and has been previously used in several studies that derive the general properties of MBPs (Keys et al. 2011 , 2014). The application of the tracking algorithm provided 300 MBPs within the observations, 449 within the $200 \mathrm{G}$ simulations, and 231 within the $50 \mathrm{G}$ simulations that we use for subsequent analysis.

We obtained magnetic field information from our spectropolarimetric data set using the NICOLE inversion algorithm (SocasNavarro et al. 2015). The inversions were run with three cycles with increasing nodes in temperature $(2,4,7)$, line-of-sight (LOS) velocity $(1,2,4)$, and $\operatorname{LOS}$ magnetic field $(1,2,3)$ between each cycle for all MBP pixels that were tracked with the process described above. In selecting the nodes for each cycle, we used a similar approach to that described in Socas-Navarro (2011). The filling factor does not vary during our inversions, but is set to 1 throughout. The initial model used was the FAL-C (Fontenla, Avrett \& Loeser 1993) quiet Sun model. Due to the small signal-to-noise ratio in Stokes $Q$ and $U$, which is expected as MBPs observed at disc centre are likely to be aligned vertically, we ignored the Stokes $Q$ and $U$ in our inversions by giving them negligible weighting within the weights file for the inversion.

To test that this is a reasonable assumption, we ran a sample of inversions including all four Stokes parameters. Again, we employed the same nodes in temperature, LOS velocity, LOS magnetic field, and then increasing nodes per cycle in the horizontal magnetic field components $\left(B_{x}, B_{y}: 1,2,3\right)$ when considering the $Q$ and $U$ profiles in our inversions. Across 20 randomly selected frames across the duration of the observations we sampled $\sim 500 \mathrm{MBP}$ pixels for inversion of all four Stokes parameters. The retrieved inclinations in 92 per cent of the cases were greater than $160^{\circ}$ (nearly perfectly vertical) with a median value of $169^{\circ}$. These values are comparable to previous work (Jafarzadeh et al. 2014). We decided not to consider the inversion of the $Q$ and $U$ profiles in our subsequent analysis, as the weak signal in $Q$ and $U$ for our observations likely meant that the inversion code would overestimate the horizontal components (Jafarzadeh et al. 2014). Also, the weak signal meant that the fits in $Q$ and $U$ were poor in comparison to those we obtained in $I$ and $V$. Given that the inclination of the MBPs is largely vertical, however, the $Q$ and $U$ profiles can be omitted without issue for the total magnetic field strength, which we are interested in here.

Furthermore, within the weights file, more weight was specified to Stokes $V$ in comparison to Stokes $I$, as the signal in Stokes $V$ is weaker than that of Stokes $I$. This is a necessary step to ensure that the Stokes $V$ profiles are fitted accurately by the inversion algorithm. The weights for Stokes $V$ were altered between cycles to improve the fit of the profiles. The weights for Stokes $V$ increased from 2 in the first two cycles to 5 in the final cycle. Examples of our synthetic fits from the inversions can be seen in Fig. 2. From the model output, we were able to gather information on various properties such as the LOS magnetic field, LOS velocity, and gas pressure as a function of optical depth for all inverted pixels.

\section{RESULTS}

We examined the parameters of MBPs as a whole to search for relationships or trends. The parameters were obtained from both the MURAM outputs from our NICOLE inversions as an average value over optical depths $(\log \tau)$ ranging from -1.5 to -0.5 . These general properties are displayed in Table 1 . Note that the symbol $\cdot 1 \div$ here denotes that the $\sigma$ boundary can be be found by multiplying or dividing the mean value by the multiplicative deviation (see Limpert et al. 2008 and Utz et al. 2013 for more indepth information on this definition and lognormal distributions). Lifetime and intensity values are given with their respective standard deviations. We average over a range of optical depths to smooth out any inaccuracies that may fall within, or at least close to, the 
(a)

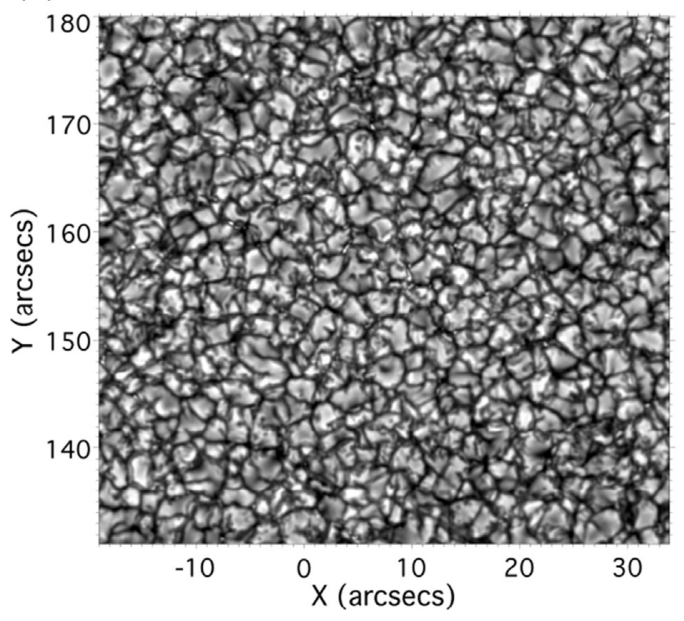

(b)

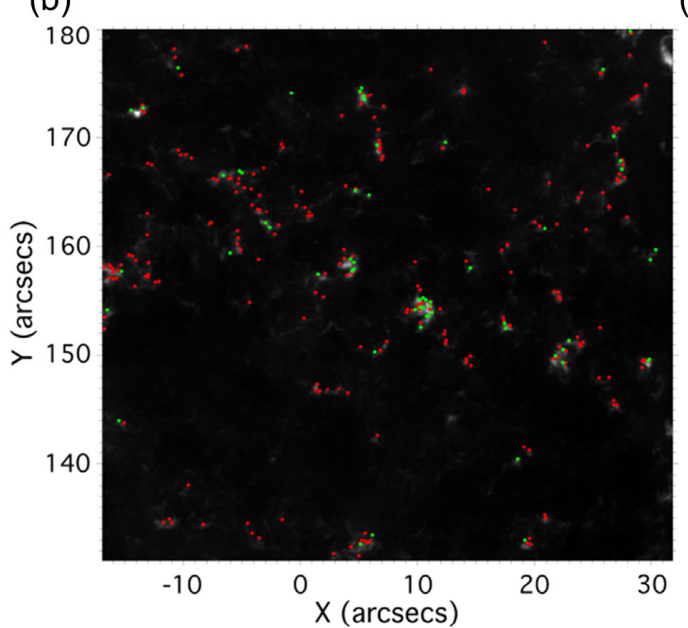

(c)

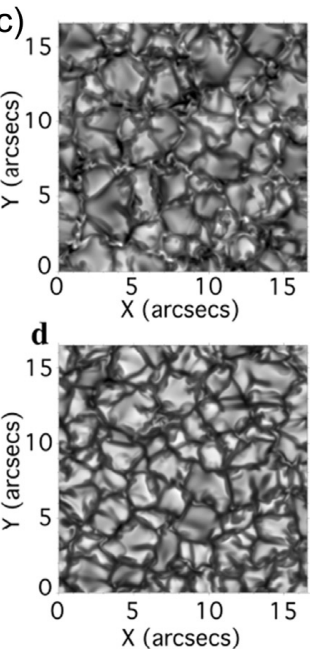

Figure 1. Panel (a) shows our observations, taken in the 6301 and $6302 \AA$ line pair with full Stokes spectropolarimetry CRISP scans at the Swedish Solar Telescope, of the quiet Sun at disc centre. The image is taken at $6301.0392 \AA$ in the line scan. Magnetic bright points can be seen in the intergranular lanes. Panel (b) shows the average location of the strong ( green) and weak (red) magnetic bright points superimposed on a map of the total circular polarization signal for the FOV averaged over the whole duration of the observations. Panels (c) and (d) show the simulated data sets we employed for the same line pair, obtained from MURAM. Panel (c) is the simulations with an initial field of $200 \mathrm{G}$ while panel (d) shows an example snapshot for simulations with an initial $50 \mathrm{G}$ field. The spatial sampling for the observations was $0.059 \mathrm{arcsec} \mathrm{pixel}^{-1}$, while the equivalent for the simulations is $0.0345 \operatorname{arcsec~pixel}^{-1}$.
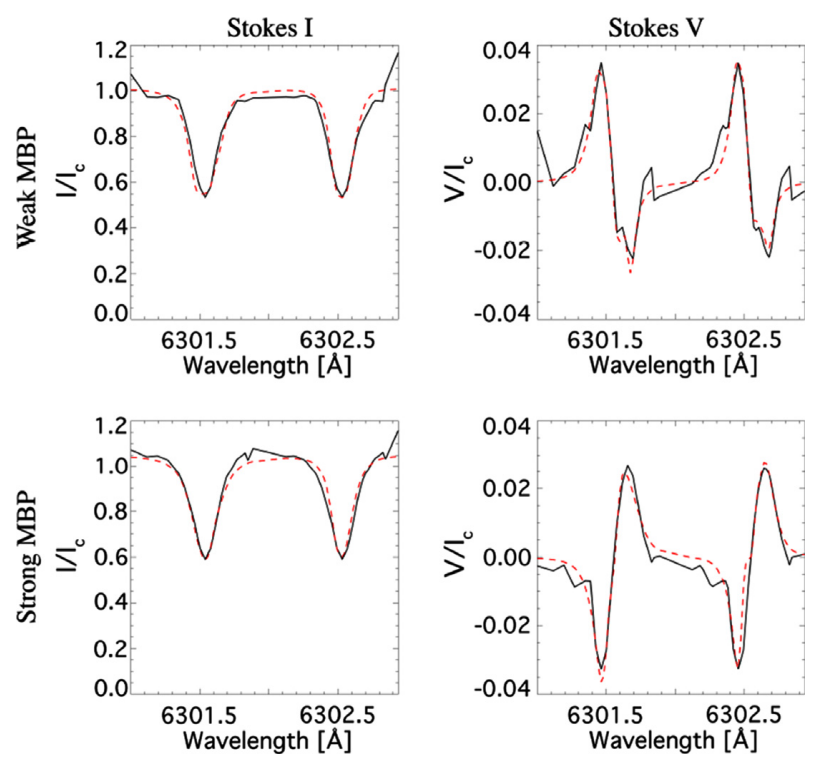

Figure 2. An example of the synthetic fits obtained from the inversion of Stokes $I$ and Stokes $V$ observations for a weak and strong MBP. In all plots the black line shows the observed Stokes $I$ or $V$ profile, while the red dashed line shows the synthetic fit after inversion with NICOLE. The left-hand column shows the Stokes I profile for a weak MBP (top) and a strong MBP (bottom). The right-hand column shows the Stokes $V$ profiles for the same two MBPs. The plots are taken for a random MBP in each distribution, and are plotted for a barycentre pixel for each. The inversions return accurate fits for our observations.

optical depth that the inversion code has chosen to fit the model. This removes any inconsistencies that may arise from choosing a singular optical depth. Furthermore, the range from -1.5 to -0.5 was chosen due to typical response functions for the line pair, which will peak closer to $\log \tau=-1$. Therefore, we expect the optical depth range chosen to be the region where the contribution is highest, thus giving
Table 1. Properties of MBPs from inversions of SST observations.

\begin{tabular}{lcc}
\hline Property & Weak group & Strong group \\
\hline Number of MBPs & 236 & 64 \\
Av. of initial $B$-field (G) & $510 \cdot \mid \div 1.6$ & $840 \cdot \mid \div 1.6$ \\
Av. of final $B$-field (G) & $530 \cdot \mid \div 1.5$ & $900 \cdot \mid \div 1.6$ \\
Av. of max. $B$-field (G) & $540 \cdot \mid \div 1.6$ & $1700 \cdot \mid \div 1.2$ \\
Av. of lifetime mean MBP $B$-field (G) & $530 \cdot \mid \div 1.4$ & $950 \cdot \mid \div 1.3$ \\
Av. lifetime & $140 \pm 60$ & $370 \pm 330$ \\
Av. of max. intensity (normalized units) & $1.07 \pm 0.03$ & $1.10 \pm 0.04$ \\
\hline
\end{tabular}

a more accurate representation of the MBPs. This likely corresponds to the mid-photosphere.

We investigated the general magnetic field strength distribution for tracked MBPs in two ways. We examined the maximum magnetic field strength detected in each MBP during its lifetime and the magnetic field distribution for all detected MBP pixels within our data sets. Fig. 3 shows the LOS magnetic field for the observations and the simulations. Column (a) shows the LOS $B$-field distribution for all MBP pixels, while column (b) shows the $B$-field distributions for the maximum $B$-field found with each tracked MBP throughout its entire lifetime. The rows show the distributions for the observations (top), the $200 \mathrm{G}$ simulations (middle), and the $50 \mathrm{G}$ simulations (bottom).

The $B$-field values derived from our spectropolarimetric inversions show two distinct groups. This distribution is similar to the one reported in Utz et al. (2013) and can be described with a double peak, defined by lognormal components. The double-peak nature of the distribution is more pronounced when one looks exclusively at the plot for the maximum $B$-field value for the $300 \mathrm{MBPs}$ over their lifetime (top right plot), with the 'weak' group peaking at $\sim 480 \mathrm{G}$ and the 'strong' group peaking at $\sim 1700 \mathrm{G}$. We note that the peak in the strong group is likely to be somewhat overestimated due to a correction factor that had to be applied to all the polarimetric calibrations of SST data taken during the 2014 observing season (see Henriques et al. 2017 for more details). This effect is non- 
(a)
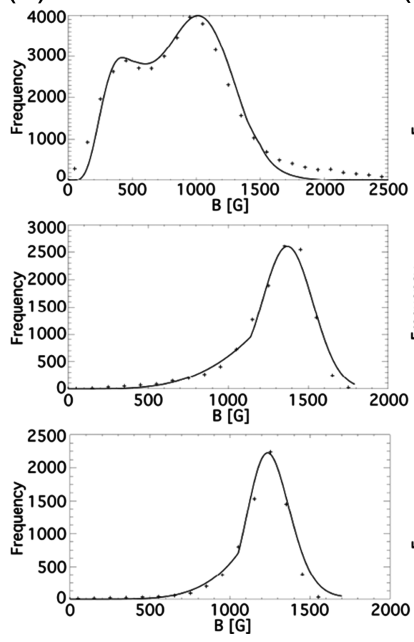

(b)
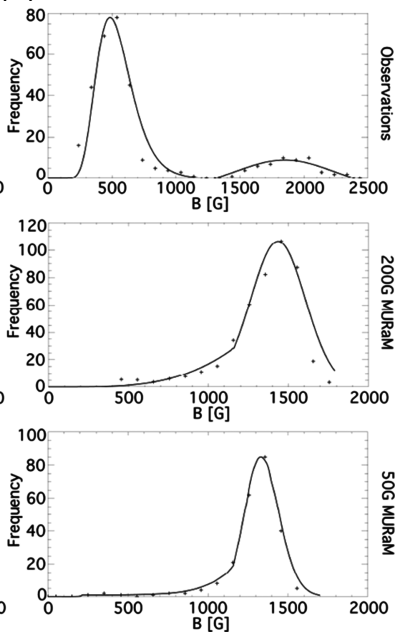

Figure 3. $B$-field properties of the MBPs from the SST observations, $200 \mathrm{G}$ MURAM simulations and $50 \mathrm{G}$ MURAM simulations. Column (a) shows the $B$ field distribution of all tracked MBP pixels in the data. Column (b) shows the $B$-field distribution for the maximum $B$-field for each tracked MBP across its lifespan. A bimodal distribution is seen in the observations, but is not recreated in either the $200 \mathrm{G}$ or $50 \mathrm{G}$ simulations. Note that the distributions are displayed for the entire duration of the observations and simulations. In the case of the simulations, this represents the distribution after the field has been injected and allowed to stabilize. All distributions are fitted with a double lognormal distribution, similar to the approach adopted by Utz et al. (2013).

linear and is more pronounced at smaller $B$-field values, though we stress that the strong group fields will still peak above the $\sim 1300 \mathrm{G}$ equipartition field strength suggested by Spruit (1979).

The bimodal distribution is less pronounced when considering all MBP pixel values. This has the effect of moving the strong peak closer (now at $\sim 1100 \mathrm{G}$ ) to the weak peak. This is not surprising as pixels about the barycentre of the MBP are likely to have similar LOS magnetic fields to the barycentre (where we expect the maximum peak for the MBP to be) and, therefore, will contribute more to the distribution, albeit lowering the location of the peak. Also, it is worth noting that it is expected that the magnetic field within each MBP would smoothly decline in strength from the strongest central value towards the MBP boundary, so a more continuous distribution would likely form when considering all MBP pixels.

Considering only the maximum $B$-field values, the average field strength of the weak group is $540 \cdot \mid \div 1.6 \mathrm{G}$, while the strong group has an average $B$-field of $1700 \cdot 1 \div 1.2 \mathrm{G}$.

A bimodal distribution is not apparent in MURAM simulations. The simulated data sets show a single peak that corresponds to the 'strong' group in our observations with no corresponding 'weak' group peak. Similar to the observations, the distribution can be fitted with a lognormal, albeit with quite a sharp tail at about $1100 \mathrm{G}$. The smaller average field strength introduced in the domain results in a smaller number of MBPs being detected in the $50 \mathrm{G}$ simulations than in the $200 \mathrm{G}$ simulations. The peak in the $B$-field distribution is slightly lower in the $50 \mathrm{G}$ simulations (peaking about $1300 \mathrm{G}$ as opposed to $1400 \mathrm{G}$ for the $200 \mathrm{G}$ simulations). This is coupled with a narrower lognormal distribution in the $50 \mathrm{G}$ simulations in comparison with the $200 \mathrm{G}$ simulations.

The absence of the two-peaked distribution in the simulations may not be completely unexpected. When considering the sim- ulations, one has to bear in mind that the simulated domain of the simulated box is small compared to the observations. Solar phenomena, such as supergranulation, are therefore missing from the simulations and that can affect the bulk motion and/or the $B$ field distributions of MBPs. This is in part why we looked at two simulated data sets to assess if different average magnetic field strengths, which we might see between network and internetwork regions, result in the different $B$-field distributions. This does not seem to be the case. In fact, when we used local correlation tracking (LCT; November \& Simon 1988) of long-duration Helioseismic and Magnetic Imager (HMI; Schou et al. 2012) continuum images to track supergranular evolution (similar to the approach employed by Requerey et al. 2018) within our FOV, we did not see a correlation between the MBP positions within the network and the maximum magnetic field strength during their evolution. Panel (b) of Fig. 1 shows the spatial location of the strong and weak MBPs within the FOV.

We believe that the most likely explanation for the lack of a two peaked distribution in the simulations arises as a result of the effects of diffusion and the fact that no 'new' flux is added to the simulated domain as a function of time. In terms of the diffusion within the simulated domain, the process is artificial, requiring additional terms to diffuse the field. Therefore, the domain becomes more 'stable' and the flux concentrations have the time to make it to kilogauss fields.

Using the methods described in Abramenko et al. (2011), we established the diffusion index $(\gamma)$ and the diffusion coefficient $(K)$ for our data and simulations. For the observations, we find values for $\gamma$ of $1.73 \pm 0.25$ for the weak MBP distribution and $1.40 \pm 0.15$ for the strong MBP distribution. These values suggest that the MBP motion for both weak and strong MBPs is super diffusive, with similar values to other studies (Keys et al. 2014). The strong MBPs are slightly less super diffusive due to clustering in regions of higher average flux (see Fig. 1, panel b) and, therefore, fall within stagnation points that restrict movement. This is reflected in the values for $K$ for the two groups of $162 \pm 46$ and $143 \pm 48 \mathrm{~km}^{2} \mathrm{~s}^{-1}$ for the weak and strong distributions, respectively, where $K$ indicates the efficiency of the dispersion of the MBPs.

The simulations were found to have values for $\gamma$ of $1.63 \pm 0.47$ and $1.77 \pm 0.54$ for the 200 and $50 \mathrm{G}$ simulations, respectively. Both sets of simulations have similar diffusive properties to those of the weak group in our observations. The value for $K$ for the simulations was found to be $160 \pm 48$ and $166 \pm 49 \mathrm{~km}^{2} \mathrm{~s}^{-1}$ for the 200 and $50 \mathrm{G}$ simulations, respectively. This seems to be slightly higher than their strong MBP counterparts in the observations, which suggests that the diffusion within the simulations is not quite the same as those observed on the Sun, with fewer stagnation points.

Given the similarity between the simulations and the weak group in terms of diffusion, it would seem that there is another reason that accounts for the difference between the two distributions. The magnetic flux within the simulated domain remains static, in the sense that no fresh flux is added over time. In contrast, the new flux can be added to our observational field of view, contributing to the generation of new MBPs with weaker fields which are being dispersed through turbulence prior to reaching kilogauss strengths, which is reflected in the values for $\gamma$ and $K$ that we find in our observations (i.e. the weak MBPs are more diffusive). In the simulated domain, the flux remains within the relatively small box and is able to form kilogauss concentrations over time. An examination of the weak to strong MBP number ratio in the simulations shows that it drops to $1: 5$ from $2: 1$ within $340 \mathrm{~s}$ in the $50 \mathrm{G}$ simulations and from $4: 5$ to $1: 5$ in $357 \mathrm{~s}$ in the $200 \mathrm{G}$ 
simulations. This supports the idea that the absence of new flux within the simulated domain results in the disappearance of the weak group and allows the MBPs to form kilogauss fields. This highlights the importance of flux emergence and diffusion of this new flux in MBP $B$-field distributions.

This double-peaked distribution has sometimes been attributed to the process of convective collapse (Utz et al. 2013), with the weak field distribution attributed to uncollapsed fields. The process of convective collapse implies a correlation between the LOS $B$ field and the LOS downflow velocity experienced by the MBPs throughout their lifetimes. Like the LOS $B$-field, the LOS velocity is sampled over the same optical depth range. Response functions for the LOS velocity suggest that there are significant contributions within this height range, with more contribution between the range $\log \tau=0$ to -1 than observed for response functions of the LOS $B$-field. We choose the same range of optical depths as the LOS $B$ field so that they are more readily comparable. We note from tests of the variation of LOS velocity that in deeper regions the values are slightly higher in magnitude, though the trends in the rise/fall of LOS velocity are comparable between the region we sample and deeper regions. A Spearman's rank correlation coefficient (SRCC) between the LOS $B$-field and the preceding LOS velocity for the strong MBPs in our observations gives a value of 0.202 , a very weak linear trend between the two parameters. The SRCC for the weak group is 0.206 , which is a similar relationship. We note that preceding here refers to a time frame of $297 \mathrm{~s}$ before (and including) the peak $B$-field. We include the frame of the peak $B$-field here in case the peaks in both parameters are coincident due to the temporal resolution. These values suggest that there is no real correlation between the magnetic field strength and the preceding LOS velocity, or it is very weak at best. It should be noted as well, however, that it cannot be discounted that convective collapse could occur on a timescale shorter than our scan time of $33 \mathrm{~s}$, although a large number of studies of convective collapse have comparable temporal resolution and find time-scales for collapse of the order of minutes (Nagata et al. 2008; Fischer et al. 2009), which would be sufficiently sampled by our temporal resolution. Furthermore, it should be noted that counter streaming flows (as observed by Bellot Rubio et al. 2001 and Utz et al. 2014, for example) could further complicate any treatment of LOS velocities, although by considering preceding values, we expect to miss the rebound shocks described by Bellot Rubio et al. (2001).

Similar values were observed when applied to our simulations $(\mathrm{SRCC}=0.115$ for $200 \mathrm{G}$ and -0.239 for $50 \mathrm{G})$. These values suggest that there is a very weak (at best) linear trend between the magnetic field and the preceding LOS velocity. Now, this does not necessarily suggest that convective collapse does not occur, only that the strength of the $B$-field is not reliant on the strength of the preceding downflow. Given the fact that strong downflows are required for collapse to occur, these values suggest that convective collapse is not solely responsible for the stronger $B$-fields in the MBPs and, therefore, it is not solely responsible for the bimodal distribution of magnetic field strengths as was once thought. Work by Utz et al. (2014) comes to similar conclusions, in that it seems that other processes besides convective collapse can result in kilogauss fields in MBPs. Within this work the authors study a large sample of MBPs and find evidence for kilogauss fields without the telltale signatures of convective collapse. The results from our study of the simulations suggest that flux emergence and diffusion of new flux play a significant role in this distribution and the ability for MBPs to form kilogauss structures.

\section{CONCLUSIONS}

In this study we analysed high-resolution spectropolarimetric data of a quiet Sun region at disc centre to examine the general magnetic properties of magnetic bright points. We find a bimodal distribution of magnetic field strengths in MBPs. We do not find significant evidence that this distribution results from the process of convective collapse in MBPs. Furthermore, the spatial locations of MBPs within network cells do not seem to explain this bimodal distribution in terms of $B$-field strength; i.e. the distribution does not appear to arise based on whether the MBPs are classed as network or internetwork MBPs.

We examined MURAM simulations to search for evidence of the bimodal distribution in simulated data sets. We investigated two sets of simulations, one with an initial field of $200 \mathrm{G}$ and one with an initial field of $50 \mathrm{G}$. However, neither returns the bimodal distribution that we see in the observations. On closer inspection, we determined that the simulated MBPs had similar diffusive properties to those of the weak MBP distribution in our observations. However, on examining the ratio of weak to strong MBPs over time in the simulations, we see that the weak distribution rapidly declines with the number of strong MBPs increasing. We attribute this to the fact that no new flux is added to the simulated domain, so the magnetic field has more opportunity to amplify to kilogauss field strengths. Within the observations, new flux is constantly added, which disperses prior to reaching kilogauss fields, thus producing the bimodal distribution we observe.

The evidence that we find in our observations and simulations suggests that this bimodal distribution is not solely the result of the convective collapse process. We see that diffusion and emergence of new flux play a significant role in the distribution of magnetic fields at these scales on the solar surface.

\section{ACKNOWLEDGEMENTS}

All authors are grateful to the anonymous referee whose comments enhanced the manuscript. PHK is grateful to theLeverhulme Trust for the award of an Early Career Fellowship. PHK would like to thank Drs. David Kuridze and Hector Socas-Navarro for assistance in setting up the inversion codes. VMJH was supported by the Research Council of Norway, project number 250810. Both VMJH and SJ were supported by the Research Council of Norway through its Centres of Excellence scheme, project number 262622. SJ also acknowledges support from the European Research Council (ERC) under the European Union's Horizon 2020 research and innovation program (grant agreement No. 682462). DBJ wishes to thank the UK Science and Technology Facilities Council (STFC) for the award of an Ernest Rutherford Fellowship alongside a dedicated Research Grant. DBJ also wishes to thank Invest NI and Randox Laboratories Ltd. for the award of a Research \& Development Grant (059RDEN1) that allowed this work to be undertaken. This research was undertaken with the assistance of resources and services from the National Computational Infrastructure (NCI), which is supported by the Australian Government. This work used the DiRAC Data Centric system at Durham University, operated by the Institute for Computational Cosmology on behalf of the STFC DiRAC HPC Facility. DiRAC is part of the UK National E-Infrastructure. Observations were acquired within the SolarNet Project Transnational Access Scheme. SolarNet is a project supported by the EUFP7 under Grant Agreement 312495. The SST is operated on the island of La Palma by the Institute for Solar Physics of Stockholm 
University in the Spanish Observatorio del Roque de los Muchachos of the Instituto de Astrofísica de Canarias. We acknowledge support from the STFC Consolidated Grant to Queen's University Belfast.

\section{REFERENCES}

Abramenko V. I., Carbone V., Yurchyshyn V., Goode P. R., Stein R. F., Lepreti F., Capparelli V., Vecchio A., 2011, ApJ, 743, 133

Bellot Rubio L. R., Rodríguez Hidalgo I., Collados M., Khomenko E., Ruiz Cobo B., 2001, ApJ, 560, 1010

Cegla H. M., Shelyag S., Watson C. A., Mathioudakis M., 2013, ApJ, 763 , 95

Crockett P. J., Mathioudakis M., Jess D. B., Shelyag S., Keenan F. P., Christian D. J., 2010, ApJ, 722, L188

de la Cruz Rodríguez J., Löfdahl M. G., Sütterlin P., Hillberg T., Rouppe van der Voort L., 2015, A\&A, 573, A40

Dunn R. B., Zirker J. B., 1973, Sol. Phys., 33, 281

Fischer C. E., de Wijn A. G., Centeno R., Lites B. W., Keller C. U., 2009, A\&A, 504, 583

Fontenla J. M., Avrett E. H., Loeser R., 1993, ApJ, 406, 319

Henriques V. M. J., 2012, A\&A, 548, A114

Henriques V. M. J., Mathioudakis M., Socas-Navarro H., de la Cruz Rodríguez J., 2017, ApJ, 845, 102

Jafarzadeh S., Solanki S. K., Lagg A., Bellot Rubio L. R., van Noort M., Feller A., Danilovic S., 2014, A\&A, 569, A105

Keys P. H., Mathoiudakis M., Jess D. B., Shelyag S., Crockett P. J., Christian D. J., Keenan F. P., 2011, ApJ, 740, L40

Keys P. H., Mathioudakis M., Jess D. B., Shelyag S., Christian D. J., Keenan F. P., 2013, MNRAS, 428, 3220

Keys P. H., Mathioudakis M., Jess D. B., Mackay D. H., Keenan F. P., 2014, A\&A, 566, A99
Leighton R. B., 1963, ARA\&A, 1, 19

Limpert E., Stahel W. A., Abbt M., 2008, BioScience, 51, 341

Nagata S. et al., 2008, ApJ, 677, L145

November L. J., Simon G. W., 1988, ApJ, 333, 427

Parker E. N., 1978, ApJ, 221, 368

Requerey I. S., Cobo B. R., Gošić M., Bellot Rubio L. R., 2018, A\&A, 610, A84

Scharmer G. B., 2006, A\&A, 447, 111

Scharmer G. B., Bjelksjo K., Korhonen T. K., Lindberg B., Petterson B., 2003, in Keil S. L., Avakyan S. V., eds, Proc. SPIE Conf. Ser. Vol. 4853, Innovative Telescopes and Instrumentation for Solar Astrophysics. SPIE, Bellingham, p. 341

Scharmer G. B. et al., 2008, ApJ, 689, L69

Schou J. et al., 2012, Sol. Phys., 275, 229

Socas-Navarro H., 2011, A\&A, 529, A37

Socas-Navarro H., de la Cruz Rodríguez J., Asensio Ramos A., Trujillo Bueno J., Ruiz Cobo B., 2015, A\&A, 577, A7

Spruit H. C., 1979, Sol. Phys., 61, 363

Utz D., Jurčák J., Hanslmeier A., Muller R., Veronig A., Kühner O., 2013, A\&A, 554, A65

Utz D., del Toro Iniesta J. C., Bellot Rubio L. R., Jurčák J., Martínez Pillet V., Solanki S. K., Schmidt W., 2014, ApJ, 796, 79

van Noort M., Rouppe van der Voort L., Löfdahl M. G., 2005, Sol. Phys., 228, 191

Vögler A., Shelyag S., Schüssler M., Cattaneo F., Emonet T., Linde T., 2005, A\&A, 429, 335

Webb A. R., Roberts B., 1978, Sol. Phys., 59, 249

This paper has been typeset from a $\mathrm{T}_{\mathrm{E}} \mathrm{X} / \mathrm{L} \mathrm{T} \mathrm{E} \mathrm{X}$ file prepared by the author. 